\title{
Competencias Docentes y Transformaciones en la Educación en la Era Digital
}

\author{
Sandra Villarreal-Villa( ${ }^{(1)}$, Jesús García-Guliany ${ }^{(2)}$, Hugo Hernández-Palma ${ }^{(3)}$, y Ernesto Steffens-Sanabria ${ }^{(4)}$ \\ (1) Universidad del Norte, Facultad de Educación, Km.5 Vía Puerto Colombia, Barranquilla-Colombia. \\ (e-mail: sandravillareal@uninorte.edu.co) \\ (2) Universidad Simón Bolívar, Fac. Administración y Negocios, Calle 59 \#59-81. Barranquilla-Colombia. \\ (e-mail: Jesus.garcia@unisimonbolivar.edu.co) \\ (3) Universidad del Atlántico, Fac. Ciencias Económicas, Carrera 30 \# 8- 49. Puerto Colombia -Colombia. \\ (e-mail: hugohernandezp@mail.uniatlantico.edu.co) \\ (4) Corporación Universitaria Latinoamericana, Fac. Ciencias Económicas, Calle 58 \# 55-24A Barranquilla-Colombia \\ (e-mail: steffensse@ul.edu.co)
}

Recibido Mar. 8, 2019; Aceptado Abr. 23, 2019; Versión final Jun. 5, 2019, Publicado Dic. 2019

\begin{abstract}
Resumen
Se analizan aspectos relacionados con las competencias digitales en docentes en el escenario de educación superior en Barranquilla, Colombia. Se realizó una investigación con un enfoque mixto, que aplicando herramientas cuantitativas y cualitativas con el apoyo de un instrumento tipo Likert abordó el tema con una muestra dirigida (intencionada) de 20 docentes. Se revisó la literatura para identificar los modelos institucionales más acogidos para la incorporación de las tecnologías de la información y comunicaciones en los ambientes académicos y se contrastó con los resultados obtenidos. Los resultados evidencian una alta autopercepción (superior al $80 \%$ ) por parte del profesorado encuestado frente a la presencia de competencias digitales en los procesos de enseñanza y aprendizaje. Entre estas, destacaron la capacitación e información, aplicación de las tecnologías de la información y la comunicación y la gestión de entornos de aprendizaje. SE concluye que se debe promover en los docentes mayor compromiso con la renovación del conocimiento y la información, así como la estimular la creación de contenido digital que fomente las competencias en el alumnado.
\end{abstract}

\section{Teacher Competences and Transformations in Education in the Digital Age}

\begin{abstract}
Aspects related to digital competencies in teachers in the higher education scenario in Barranquilla, Colombia, are analyzed. A research was carried out with a mixed approach, applying quantitative and qualitative tools with the support of a Likert type instrument and addressing the topic with a targeted (intentional) sample of 20 teachers. The literature was reviewed to identify the most accepted institutional models for the incorporation of information and communication technologies in academic environments, and the results obtained were compared. The results show a high level of self-perception (over $80 \%$ ) by the teachers surveyed in view of the presence of digital competences in teaching and learning processes. Among these, training and information, the application of information and communication technologies and the management of learning environments stand out. It is concluded that it is necessary to promote in teachers greater commitment to the renewal of knowledge and information, as well as to stimulate the creation of digital content that promotes the skills in students.
\end{abstract}

Keywords: information and communication technologies; digital skills; teacher's competence; higher education 


\section{INTRODUCCIÓN}

En la actualidad, las tecnologías de la información y la comunicación (TIC) se han abierto camino en la industria, la comunicación, la educación, el trabajo y hasta la recreación. Los desafíos no solo existen en la adquisición y adopción de tecnología de la información y la comunicación (TIC), sino también en la tarea más compleja de utilizar recursos tecnológicos para facilitar de manera efectiva la innovación y la mejora educativas (Thomas y Knezek, 2008). Uno de los objetivos más desafiantes de la educación de la actualidad es capacitar a los niños y jóvenes para enfrentar los diferentes retos que supone el mundo actual (Tyner (2014); de ahí que uno de los elementos más influyentes en los diferentes ámbitos de la vida humana son las nuevas tecnologías de la información y comunicación (TIC). Las TIC han cambiado en su totalidad la forma de gestionar negocios, servicios, la cultura y las relaciones humanas; sin embargo, aún su potencial no ha sido desarrollado del todo en muchos escenarios. Las TIC pueden ser de gran ayuda para el acceso universal al conocimiento, derivando en aspectos como equidad, desarrollo profesional y crecimiento económico (Venkatesh, et al., 2014).

A nivel internacional se identifican varios modelos y marcos conceptuales que han intentado definir la Competencias Digitales Docentes (CDD) (Esteve, 2015). En la actualidad se han difundido varios estudios que efectúan análisis y contrastan dichos marcos, cada uno de ellos ofrece diversas alternativas aplicables según cada escenario o panorama de educación (Van Laar, et al., 2017). Independientemente de las desigualdades entre los diferentes modelos, procedentes en principio del contexto, del entorno educativo 0 del designio con el que fueron diseñados; generalmente todos ellos se originan de la tecnología y de los cambios que esta representa para la humanidad o para los sistemas productivos particularmente $\mathrm{y}$, sobre ello, se establece una capa de enseñanza que redirige el accionar en el ámbito de educación y sus distintos niveles. Esa capa en una situación ideal está fundamentada en una concepción básica de la educación, siendo reducida a la acción en el aula en la que, en una situación ideal, el rol del docente suele restringirse al de formador individual de un alumno en colectividad. Dentro del análisis planteado se hace referencia a los estándares internacionales de mayor uso y transcendencia en la formación de competencias de los documentos frente a la utilización de las nuevas tecnologías de la información y la comunicación, es así como se observó la utilidad de los estándares relacionados con el tema, de la International Society for Technology in Education, los de la UNESCO y otros aportes al proceso educativo.

La sociedad actual se caracteriza por su cambio tecnológico constante, los alumnos de hoy cada día están más sensibilizados con el mundo de las tecnologías; es por ello que los educadores se ven en la necesidad de aprovechar esta nueva fuente de motivación intrínseca utilizando herramientas de este tipo e incorporarlas en el desarrollo de actividades que animen a los alumnos a tomar parte activa en sus procesos de aprendizaje. El uso de estos mecanismos de enseñanza permite aumentar la posibilidad de promover nuevas formas de aprendizaje orientados al desarrollo de estrategias de pensamiento y la interacción con otros individuos mientras se estimula su nivel de actividad personal (Stošić, 2015). El uso de tecnologías permite que la información sea adquirida, procesada, almacenada y diseminada, lo que contribuye a la capacitación de las personas para que puedan adaptarse a los nuevos desafíos sociales. En este sentido, se considera que la formación del educador debe estar orientada a la generación de reflexiones en procesos destinados a la promoción del uso pedagógico de herramientas, recursos, programas, servicios y entornos caracterizados por el uso de la tecnología (Hepp, et al., 2015).

\section{MODELOS SOBRE COMPETENCIAS DIGITALES DOCENTES}

Propuesto por la International Society for Technology in Education (ISTE), el National Educational Technology Standards for Teachers (NETS-T), internacionalmente es uno de los modelos con más incidencia; dicho estándar se conforma por cinco dimensiones que se generan dependiendo del desempeño de los docentes (figura 1), de igual forma está focalizado en la forma en que los docentes pueden hacer más sencillo el aprendizaje de los alumnos a través de las TIC, abarcando todos los aspectos (ciudadanía digital y gestión de materiales), incluyendo el desarrollo profesional del pedagogo. Este modelo ha sido focalizado, mediante la reciente actualización del 2017, a diferencia de su versión anterior del 2008, en relación a la trascendencia de que los alumnos sean autodidactas y gestionen su propio aprendizaje. Para lograr ese objetivo hay una serie de características predeterminadas para que el docente esté capacitado para ser el "catalizador" del aprendizaje (ISTE, 2018).

Según el ISTE (2018), el objetivo principal del estándar NETS-T, es establecer los lineamientos para que los educadores diseñen, implementen y evalúen experiencias educativas para involucrar a los estudiantes y mejorar el aprendizaje, enriquecer la práctica profesional, y proporcionar modelos positivos por todos los actores del contexto educativo (ISTE, 2018); en este sentido se explican los indicadores de representados en la figura 1 ; 1) Facilitar e inspirar el aprendizaje y la creatividad del estudiante: se promueve el uso del conocimiento de la materia, procesos de enseñanza y tecnología para facilitar el aprendizaje de los 
estudiantes, la creatividad e innovación en entornos virtuales; 2) Diseñar y desarrollar experiencias y asesorías de aprendizaje en la nueva era digital: se establece el diseño, desarrollo y evaluación de experiencias de aprendizaje mediante la incorporación de herramientas y recursos contemporáneos; 3) Modelar el trabajo y aprendizaje en la nueva era digital: los maestros deben exhibir conocimientos, habilidades y procesos de trabajo característicos de un profesional innovador en una sociedad global y digital; 4) Promover y modelar la ciudadanía y responsabilidad digital: se busca la comprensión de los recursos de las sociedades locales y globales, así como también la responsabilidad de incorporar la cultura digital a través de un comportamiento ético y legal en sus prácticas profesionales; 5) Participar en el crecimiento profesional y el liderazgo: se estimula el mejoramiento de la práctica profesional de los maestros, mediante un crecimiento permanente y la exhibición de liderazgo y en la escuela y la comunidad a la que pertenecen, promoviendo el uso efectivo de los recursos digitales.

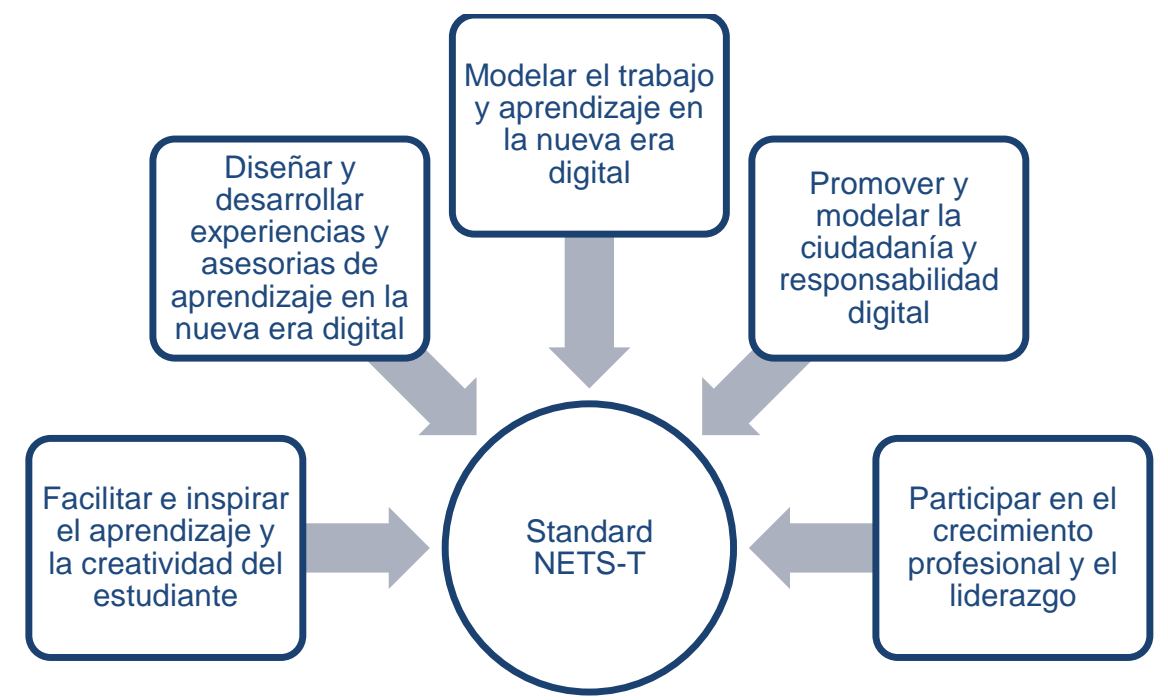

Fig. 1: Indicadores de desempeño para los educadores del NETS-T. (Adaptado de ISTE, 2018)

Otro de los modelos establece una serie de competencias estandarizadas en TIC para docentes en formación y para los que ejercen la carrera, éste pertenece al grupo de los modelos más conocidos a nivel internacional por UNESCO. Un modelo con visión estrictamente organizacional, que además de tomar en cuenta las ventajas didácticas también toma en cuenta asuntos como la inclusión de las TIC en los planes de estudios o en la formación profesional de docentes. La estructura de dicho modelo se presenta en la figura 2 , y se fundamenta en el establecimiento de las prácticas pedagógicas que favorezca la construcción significativa de conocimiento de los estudiantes a través de la integración de las TIC; de tal manera que reúne las competencias necesarias para el diseño, implementación y evaluación de dichos ejercicios educativos en virtud del desarrollo de las representaciones del saber, como son el conocer, utilizar y transformar tales recursos tecnológicos.

Como se observa, el modelo de UNESCO se basa en el desarrollo de niveles de apropiación de las TIC que, a su vez, son desglosados en tres elementos con el fin de promover el buen uso de las tecnologías de la información, lo que se busca es lograr que los maestros establezcan su nivel de competencias de acuerdo a los estándares planteados, para así establecer planes de formación y crecimiento profesional en relación al tema en cuestión (Pontificia Universidad Javeriana - UNESCO, 2018). Por otra parte, en Europa, el Joint Research Centre (JRC) de la Comisión Europea (European Commission), propone el Marco Europeo para la Competencia Digital del Profesorado (DigCompEdu), representado en la figura 3, el cual se fundamenta en elementos relacionados con el entorno y compromiso profesional de los educadores, la importancia de las TIC en el proceso de enseñanza-aprendizaje o volver a los alumnos autodidactas en el desarrollo de su competencia digital como ciudadanos.

Como se observa en la figura 3, el modelo contempla diversos tipos de competencias que se relacionan con su aplicación, es así como se contemplan: el compromiso profesional, los recursos digitales, la pedagogía digital, evaluación y retroalimentación, empoderamiento, y el facilitar la competencia digital de los estudiantes; a su vez, dichos aspectos pueden ser desarrollados de acuerdo a los diferentes niveles de progresión, como son: la conciencia, la exploración, la integración, maestría, liderazgo, y por último la innovación. De acuerdo a los lineamientos planteados se busca el establecimiento de programas educativos adaptables a los diferentes ambientes de aprendizaje con el fin de lograr una apropiación adecuada de los recursos tecnológicos (European Commission, 2018). 


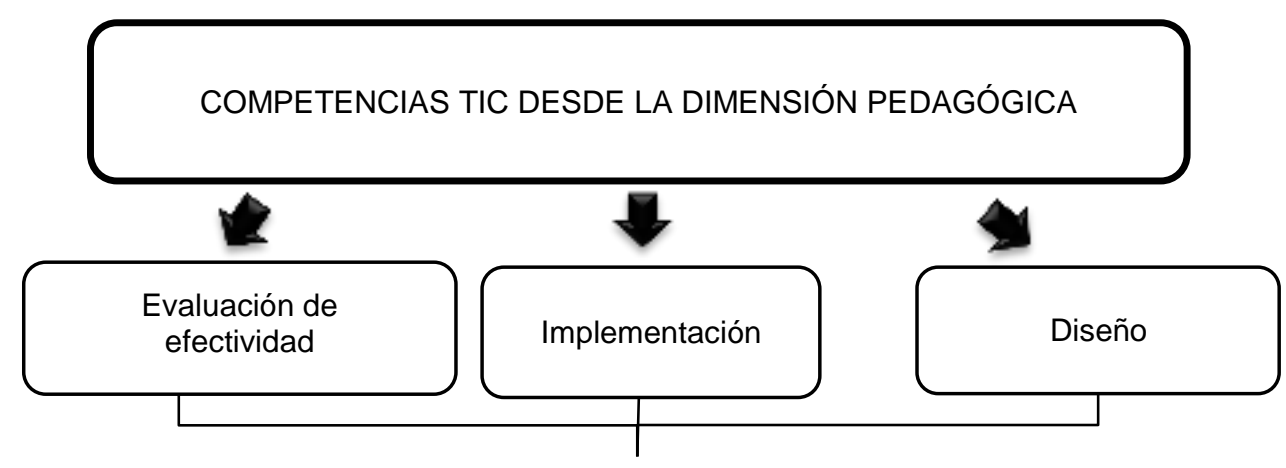

Fundamentadas en los
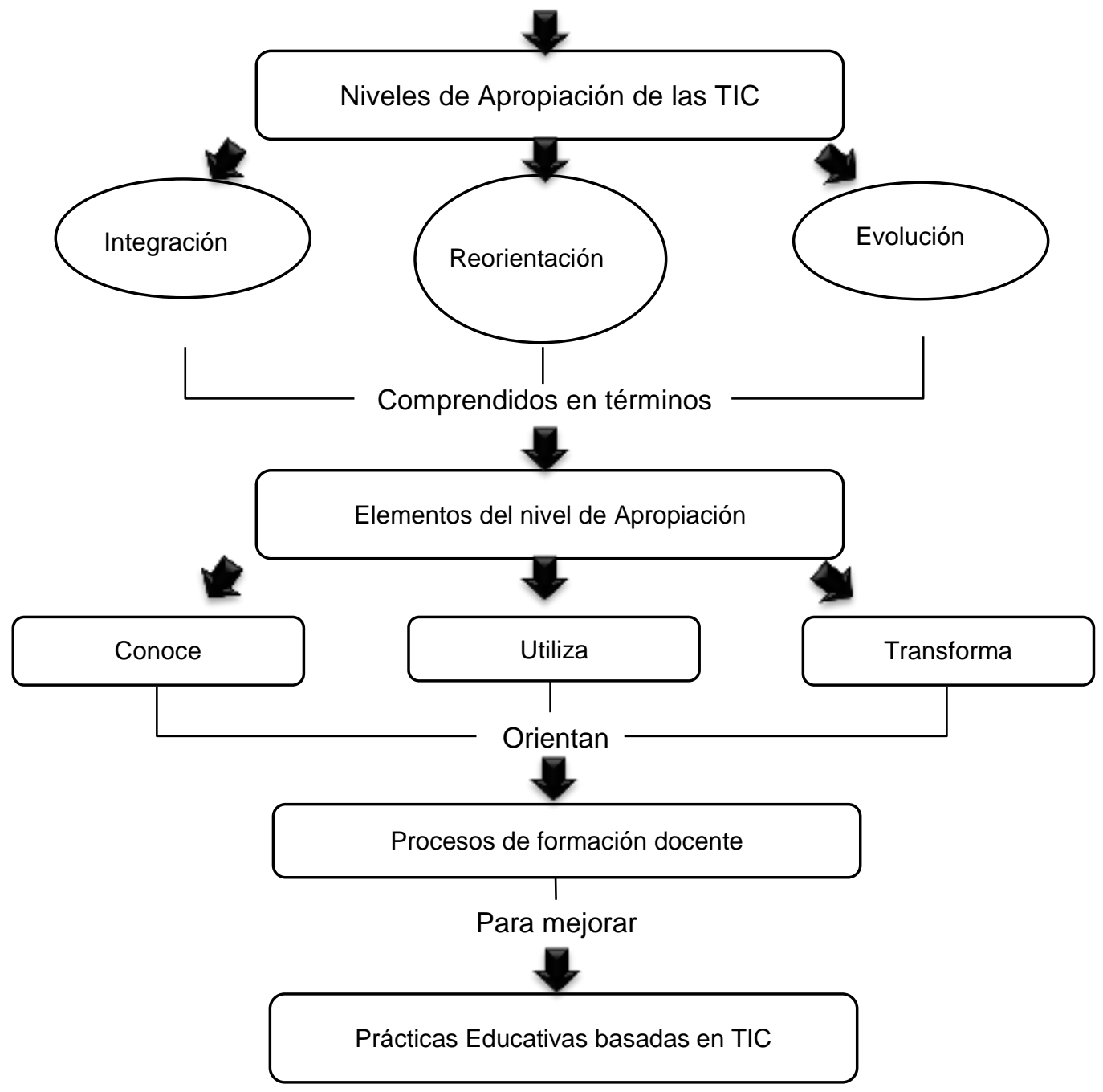

Fig. 2: Enfoque de competencias TIC en el modelo educacional de la UNESCO (Adaptada de UNESCO, 2018)

Como consecuencia de los diferentes modelos se han suscitado diferentes cambios con el paso del tiempo. Por ejemplo, en los EE. UU, basándose en el modelo NETS-T de ISTE 2008, varios estados han realizado cambios y han establecido sus propios estándares, publicando manuales y libros para guiar y facilitar su aplicación en las escuelas (Morphew, 2012). Para el diseño de planes educativos estratégicos de diferentes países o instituciones han sido tomados como base lineamientos como el de la UNESCO (2011). El modelo ISTE ha sido utilizado en varias investigaciones referentes al nivel de habilidades digitales de los docentes activos y en formación (Banister y Reinhart, 2012). 


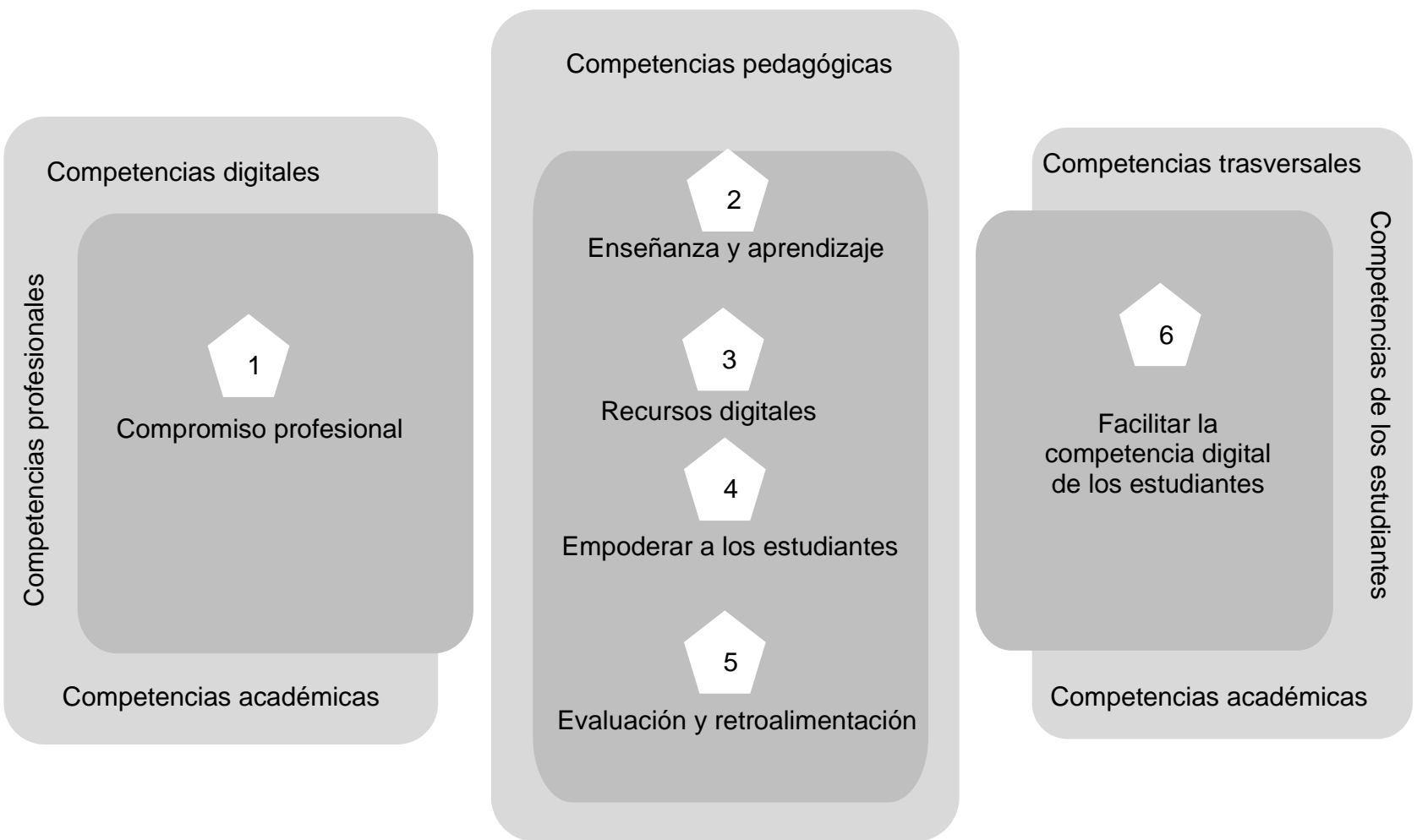

Fig. 3: Concepto de evaluación de la competencia digital en el profesorado propuesta por el JRC (Adaptado de European Commission, 2018)

\section{Nuevas alternativas TIC}

La nueva era, que es consecuencia de la evolución tecnológica en las últimas décadas, ha influido significativamente en la educación superior, no sólo porque ha mejorado los procesos administrativos, sino también porque ha abierto las puertas a nuevas modalidades de formación y espacios para el aprendizaje (Ertmer, et al., 2014; Bernard, et al., 2014; Uceda, et al., 2010). Lo antes expuesto trae como consecuencia la descentralización del conocimiento (Niebles et al., 2016), debido a que la tecnología, por su gran influencia, ha cambiado nuestras formas de hacer las actividades cotidianas, lo cual, aplicado a un entorno pedagógico, provoca que la tecnología no solo sea un medio de capacitación de los estudiantes, sino que se convierte en un entorno virtual de interacción interdisciplinario (Badilla, 2007).

Una definición balanceada de las TIC es la siguiente: Son un conjunto de técnicas desarrolladas y aplicadas a dispositivos tecnológicos que reúnen funcionalidades de procesamiento, almacenamiento y transmisión (Luz, 2018). De igual manera, en forma general se puede decir que las TIC se desarrollan principalmente en las tecnologías que tienen la cualidad de transmitir datos en cualquier lugar y momento, independientemente del periodo de tiempo en el que fue creado el aparato electrónico (Cacheiro, 2018). La integración de las TIC en el entorno educativo, tanto básico como avanzado, es un tema que está en constante debate por los múltiples puntos de vistas que hay sobre este tema, sin embargo, los esfuerzos por integrar dichas tecnologías en los entornos educativos han sido importantes en relación al objetivo que el estudiante pueda utilizarlas en su formación académica y en el ejercicio critico de su ciudadanía (Bustos y Román, 2016).

Como consecuencia de la vital importancia que han tomado las TIC, su llegada a las aulas de clase ha sido vertiginoso, en respuesta de las necesidades de la sociedad, la cual necesita que la educación vaya en la misma dirección de los procesos de transformación cultural y social generados alrededor de las TIC. Por lo antes expuesto el entorno educativo ha tenido que ser modificado en referencia al entorno administrativo y de planes de estudio para poder ser utilizados como herramientas didácticas y como herramientas de enseñanza y aprendizaje (Roblizo y Cózar, 2015). Desde el punto de vista del estudiante, las TIC deben ser herramientas útiles para la motivación y estímulo al proceso de aprendizaje (Njoku, 2015), contribuyendo al proceso de integración con la realidad, y permitiendo observar los resultados de esta interacción, el desarrollo de habilidades de pensamiento crítico y creativo, así como también el incremento de las habilidades para la retención de la información, el aprendizaje significativo y el desarrollo de competencias útiles en su desempeño en la sociedad (Gisbert y Esteve, 2016).

Por su parte, una adecuada usabilidad de las TIC hace referencia al análisis del uso de las diferentes tecnologías en su potencial de uso y aplicación en la práctica educativa (Domínguez, et al., 2015), de ahí la 
importancia de que el docente este en la capacidad de identificar los beneficios y apropiarlos adecuadamente en su aula de clases, en este sentido Colorado y Edel (2012) destacaron los siguientes beneficios del uso de las TIC en el contexto educativo: 1) Interactividad: aporta habilidades de comunicación de manera bidireccional y multidireccional en entornos virtuales, de tal manera que facilite la interrelación entre los usuarios del recurso tecnológico; 2) Aprendizaje colaborativo: facilita la formación de comunidades de usuarios en los que se desarrolla el trabajo cooperativo y colaborativo, permitiendo diversos tipos de intercambios entre los usuarios que favorecen el aprendizaje; 3) Multidireccionalidad: la capacidad de transmisión e intercambio de información que ofrecen las TIC permite el tránsito simultáneo entre múltiples destinatarios; 4) Libertad de edición y difusión: Facilita la difusión, intercambio y colaboración para la edición de contenidos entre una multitud de usuarios en la red.

Ahora bien, muchos de los profesores no tienen la formación para aplicar las herramientas de TIC y producir una metodología innovadora y enriquecedora para los alumnos. En vista de esto las Tecnologías de Aprendizaje y Conocimiento (TAC) están enfocadas más hacia el uso formativo que informativo, con la finalidad de aprender más y mejor. Es decir, que en el caso que un docente no sepa cómo implementar una herramienta de TICs, allí entra en acción las TAC, las cuales tienen como objetivo no sólo el aprendizaje del modo de utilización y control de las TICs, sino su aprovechamiento máximo para la enseñanza, formación y conocimiento (Gil, 2017). Es decir, definidas de una manera más sencilla, las TAC son herramientas de tecnología que ayudan a conseguir objetivos en el marco de los programas educativos (Expósito, Fuentes y Martínez, 2017), las cuales vienen evolucionado de las ya conocidas TIC (Vivancos, 2015).

Desde el punto de vista de Enríquez (2012), se trata de identificar y brindar la tecnología necesaria para facilitarle a los docentes la enseñanza a los alumnos, y para ambos, la habilidad de poder utilizar éstas en un entorno alejado de las instituciones educativas, de tal manera que busca favorecer el aprendizaje y aportar beneficios temporarios para un mejor procesamiento y apropiación de la información que se recibe constantemente. En este mismo orden de ideas, otro método utilizado en el entorno educativo son las Tecnologías para el Empoderamiento y la Participación (TEP) las cuales usan generalmente las redes sociales para hacer comentarios libres sobre cualquier tendencia, de forma que el público en general pueda opinar e intervenir (Pérez, et al., 2017). Igualmente se podría definir a las TEP, más sencillamente, como el conjunto de tecnologías utilizadas para fomentar la intervención de personas en un tema determinado; como consecuencia de esto se genera una consciencia ciudadana del lugar que ocupan en la sociedad (Granados, 2015).

Las TEP entonces conceden el poder de la información generada a partir de diversos puntos de vista, generando puentes entre el conocimiento individual y colectivo, propendiendo hacia una mayor participación y empoderamiento que conlleve a un aprendizaje proactivo que potencie las habilidades individuales (Peris y Lindahl, 2015), explote las inteligencias y las creatividades colectivas; todo lo anterior a través de la reunión de un gran universo de individuos sin la necesidad de compartir un mismo sitio, espacio lugar o tiempo (Meza, et al., 2017). Es evidente que con el cambiar de los tiempos también cambian las necesidades de los alumnos, los cuales se encuentran en una disyuntiva entre los requerimientos curriculares y los reales que sus entornos les demandan. En vista de esto, los docentes con el uso de las TIC, TAC y TEP deben ir más allá de la formación académica del aula, es decir, el docente no debe encajonar la educación, orientándola solo hacia el objetivo académico, por el contrario, debe gestionar la educación fuera de las aulas, despertando el sentido crítico y objetivo sobre el entorno que lo rodea (Granados, 2015); algunos de los elementos más importantes de estas se destacan en la figura 4.
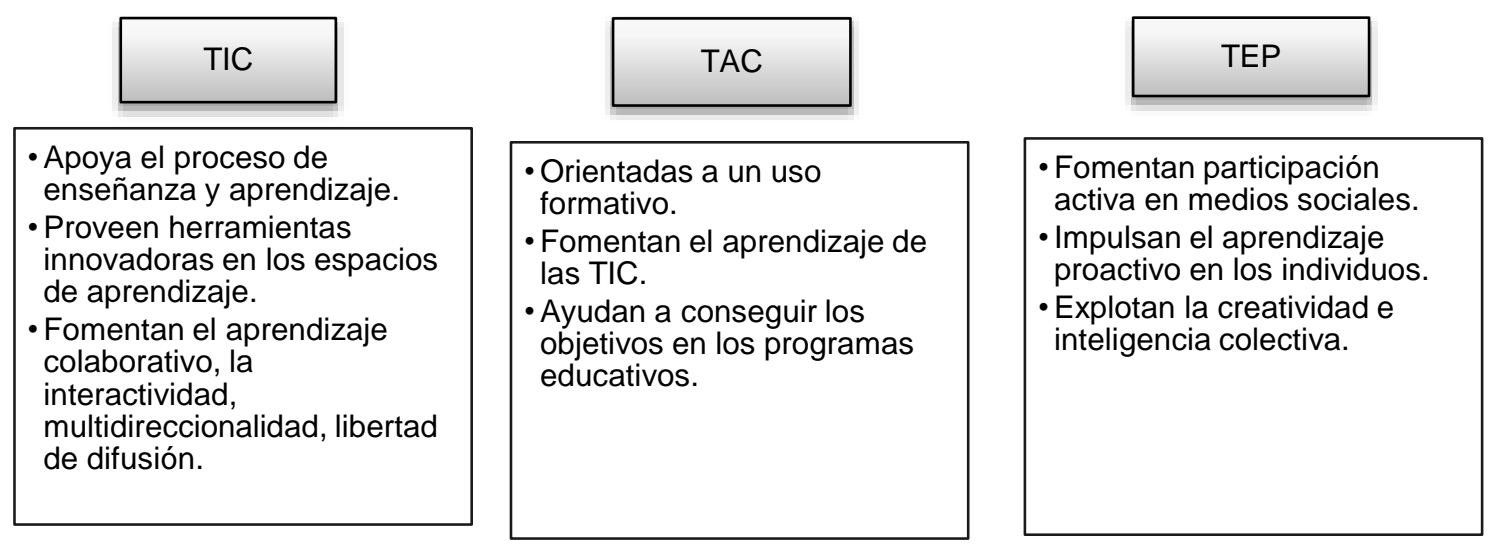

Fig. 4: Características del uso de las TIC, TAC y TEP 
Por lo antes expuesto, se puede decir entonces que el aprendizaje por medio de las TIC, TAC y TEP no solo influyen y se manejan en el entorno personal e individual del docente que va más allá de su uso instrumental (Pinto, et al., 2017), sino que también repercute en el entorno de este, debido a que el aprendizaje en comunidad implica saber interactuar, bien sea para recibir o para impartir el conocimiento, siendo el profesor el responsable de crear el entorno virtual para el aprendizaje, por lo que la tecnología tendrá un papel mediador entre la interacción social y la construcción del conocimiento (Cabero, 2014). De acuerdo al contexto globalizado, el docente y su práctica educacional deben poder ser adaptativos a los cambios del entorno (Marín et al., 2017), sabiendo que nuevas ideas surgirán constantemente, y por consiguiente la tecnología variará a este ritmo, e irá desarrollándose con muchas más herramientas que estarán al alcance de la sociedad (Granados et al., 2014), de ahí que es importante dejar en claro que el aprendizaje sobre las TIC no es el fin sino el medio para lograr un mayor alcance en el objetivo educacional de cualquier docente en la actualidad.

\section{METODOLOGÍA}

Los paradigmas orientan la investigación sobre la base de supuestos para comprender la naturaleza de la realidad, sus características y elementos que la identifican (Howard, 2016). Desde esta perspectiva, el paradigma cuantitativo contribuye a determinar las características del objeto analizado de forma cuantitativa y mesurable sin realizar intervención o influencia alguna sobre este (Gutiérrez, 2017). En función de ello, se aplica tal enfoque para diagnosticar la situación actual de modelos institucionales de competencia digital docente en universidades de la ciudad de Barranquilla. Teniendo en cuenta lo anterior, para el desarrollo del artículo se propuso un enfoque cuantitativo de tipo descriptivo, documental y de campo (Hernández, et al., 2014), que permitiera extraer la información de las competencias digitales para la construcción de una visión holística sobre la apropiación de estos modelos por parte de los docentes en las instituciones de educación superior de Barranquilla.

En primer lugar, se construyó un instrumento tipo Likert el cual estuvo conformado por 10 ítems con las principales variables relacionadas con las competencias digitales de acuerdo a la teoría revisada; el cuestionario fue validado por medio del Alfa de Cronbach, el cual arrojó un valor de 0.815 . Por otra parte, se utilizó la revisión documental de artículos científicos en bases de datos reconocidas, revistas de corriente principal, repositorios, entre otras fuentes secundarias relevantes, para recolectar información suficiente sobre la temática planteada. La población estuvo conformada por docentes de educación superior de las instituciones educativas ubicadas en la ciudad de Barranquilla, sin embargo, por razones de conveniencia y limitaciones del estudio se aplicó un muestreo dirigido intencional y no probabilístico (Otzen y Manterola, 2017) conformado por 20 docentes de las universidades más importantes de la ciudad. Asimismo, el análisis de los datos cuantitativos se apoyó en el software SPSS versión 23 utilizando tablas de frecuencia, estadísticos descriptivos básicos y tablas de soporte. A continuación, en tabla 1 se muestra las competencias evaluadas durante el proceso.

Tabla 1: Competencias Digitales y preguntas del cuestionario

\begin{tabular}{|l|l|}
\hline \multicolumn{1}{|c|}{ Competencia } & \multicolumn{1}{|c|}{ Descripción de la pregunta } \\
\hline Aplicación de las TIC & $\begin{array}{l}\text { Aplica y se apoya de forma continua en las TIC para desarrollar los procesos de } \\
\text { enseñanza. }\end{array}$ \\
\hline Capacitación e información & $\begin{array}{l}\text { Se preocupa por mantenerse informado de las últimas tendencias educativas en el } \\
\text { ámbito de las TIC. }\end{array}$ \\
\hline $\begin{array}{l}\text { Gestión de entornos de } \\
\text { aprendizaje }\end{array}$ & $\begin{array}{l}\text { Genera y gestiona de manera eficiente entornos de aprendizaje utilizando } \\
\text { herramientas tecnológicas (TIC) para fomentar la innovación y curiosidad en los } \\
\text { estudiantes. }\end{array}$ \\
\hline Promoción de las TIC & Promueve en los estudiantes el uso efectivo de herramientas y recursos digitales. \\
\hline Gestión de la información & $\begin{array}{l}\text { Integra procesos de almacenamiento y gestión de la información digital en las } \\
\text { actividades académicas. }\end{array}$ \\
\hline $\begin{array}{l}\text { Promoción del uso ético, legal } \\
\text { y seguro de las TIC }\end{array}$ & $\begin{array}{l}\text { Promueve y enseña sobre el uso seguro, legal y ético de la información digital en } \\
\text { el aula en todo momento. }\end{array}$ \\
\hline Diseño de actividades con TIC & Diseña y evalúa materiales y actividades creados en entornos digitales. \\
\hline Actividades de Innovación TIC & $\begin{array}{l}\text { Incorpora en el proceso de enseñanza actividades de innovación por medio de TIC } \\
\text { para fomentar su uso en los estudiantes. }\end{array}$ \\
\hline Ciudadanía digital & Promueve en los estudiantes la ciudadanía digital y responsable. \\
\hline $\begin{array}{l}\text { TIC para la productividad } \\
\text { profesional }\end{array}$ & Utiliza las TIC para mejorar su productividad y práctica profesional. \\
\hline
\end{tabular}




\section{RESULTADOS Y DISCUSIÓN}

Tras realizar el la tabulación y depuración de datos se procedió a analizar las variables mediante las técnicas de la estadística descriptiva. En primer lugar, para facilitar la comprensión se asoció cada pregunta a una competencia digital, de acuerdo a los modelos teóricos revisados en la literatura, en la tabla 1 se detalla la competencia relacionada con cada una de las preguntas. Seguidamente, se muestra la tabla 2 de frecuencias relativas obtenida del análisis.

Tabla 2: Competencias digitales y porcentaje de aplicación. * $\mathrm{Ns} / \mathrm{Nr}=$ No sabe/No responde

\begin{tabular}{|c|c|c|c|c|c|c|}
\hline & $\sum_{i}^{\sum}$ & 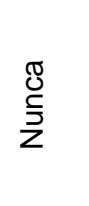 & 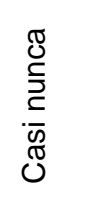 & 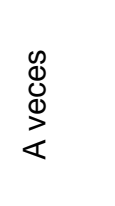 & $\begin{array}{l}\frac{0}{0} \\
\frac{0}{2} \\
\frac{0}{0} \\
\frac{0}{9} \\
0 \\
0\end{array}$ & $\begin{array}{l}\frac{0}{\overline{0}} \\
\frac{\vec{E}}{\omega} \\
\frac{\omega}{\omega}\end{array}$ \\
\hline Capacitación e información & - & - & - & $5 \%$ & $10 \%$ & $85 \%$ \\
\hline Aplicación de las TIC & $5 \%$ & - & $5 \%$ & - & $10 \%$ & $80 \%$ \\
\hline Gestión de entornos de aprendizaje & $5 \%$ & $5 \%$ & - & - & $20 \%$ & $70 \%$ \\
\hline Promoción del uso ético, legal y seguro de las TIC & $5 \%$ & - & - & $10 \%$ & $20 \%$ & $65 \%$ \\
\hline Ciudadanía digital & - & $5 \%$ & - & $10 \%$ & $20 \%$ & $65 \%$ \\
\hline Promoción de las TIC & $5 \%$ & - & $10 \%$ & $15 \%$ & $10 \%$ & $60 \%$ \\
\hline Actividades de Innovación TIC & - & $5 \%$ & $5 \%$ & $10 \%$ & $20 \%$ & $60 \%$ \\
\hline TIC para la productividad profesional & - & - & - & $20 \%$ & $25 \%$ & $55 \%$ \\
\hline Diseño de actividades con TIC & $5 \%$ & - & $10 \%$ & $30 \%$ & $10 \%$ & $45 \%$ \\
\hline Gestión de la información & & $5 \%$ & - & $25 \%$ & $40 \%$ & $30 \%$ \\
\hline
\end{tabular}

Es perceptible el alto grado de aplicabilidad, promoción y uso de las TIC que informan los docentes en el desarrollo de los procesos de enseñanza y aprendizaje. Iniciando con los porcentajes más representativos, se identifica que la capacitación e información es uno de los aspectos que más relevancia tiene para los profesionales, es decir, el $95 \%$ se preocupa activamente por mantenerse informado de las últimas tendencias educativas en el ámbito de las TIC. Al respecto, Buabeng (2012) expone que hay factores que impiden a los docentes y sistemas implementar correctamente las TIC, concretamente incluye aspectos como las falencias en las habilidades de los maestros, la poca formación pedagógica, acceso limitado y la rigidez de los sistemas tradicionales. Seguidamente, la aplicación de las TIC y la gestión de entornos de aprendizaje son un indicativo de que la mayoría de los docentes gestiona adecuadamente esos espacios para fomentar la innovación educativa. Este resultado concuerda con lo hallado en investigaciones similares como la realizada por Siddiq et al. (2016) en la cual indica que los docentes tienen una frecuencia de uso de las TIC positiva. En las competencias que se observaron algunas deficiencias, resalta la promoción del uso ético, legal y seguro de las TIC, ciudadanía digital y promoción de las TIC, donde comienzan a considerar que a veces o incluso casi nunca las aplican, en este sentido, el uso de los medios sociales en el espacio de aprendizaje tanto formal como informal podría apoyar el desarrollo de una ciudadanía digital (Gleason y Von Gillern, 2018).

Comparativamente con escenarios internacionales, Instefjord y Munthe (2017) han destacado que existe una mejora significativa entre la eficacia de los procesos de enseñanza del docente y la adquisición de competencias digitales, adicionalmente, señalan que el 35\% de los profesores llega a considerarse como buenos modelos para la incorporación de la tecnología en el ámbito educativo. Esto es coherente con los hallazgos reportados por el presente estudio, pues en la mayoría de los casos analizados los encuestados manifestaron un alto grado de acuerdo en la aplicación de las competencias tecnológicas, es decir, se podría considerar que cuentan con una auto percepción positiva en este aspecto. Por otra parte, con base en la perspectiva de Svensson y Baelo (2015) la Comisión Europea ha definido la competencia digital como uno de los componentes clave en la educación actual, enmarcándola en el uso seguro, crítico y creativo de las tecnologías con el propósito mejorar el aprendizaje, por ello, se exponen la importancia de abocarse a integrar esto elementos en los programas de formación de profesores. En este sentido, valdría la pena indagar sobre cómo los docentes logran adquirir estas competencias y si están siendo abordadas desde sus procesos formativos a nivel de educación superior.

En el escenario actual, donde la masificación de la educación es un hecho a través de las facilidades que brinda el internet y las diferentes formas de conexión, impartir conocimiento de calidad a todos los participantes y además atender a sus necesidades individuales, puede llegar a ser una actividad compleja. En este sentido, Stehling et al. (2016) argumentan que el uso de las TIC en aulas presenciales con más de 1.500 estudiantes puede afectar significativamente su aprendizaje. En virtud de ello, propusieron en la Universidad Técnica de Aquisgrán (RWTH) un entorno educativo interactivo mediante el desarrollo de un 
Sistema de Respuestas de Audiencia (SRA), lo que implicó una modificación importante en el diseño de las estructuras tradicionales de las conferencias, haciendo énfasis en el contenido. Entre los resultados obtenidos, se menciona el aumento del interés y la atención de los estudiantes, dado que para el docente esta labor llegaba a ser agotadora atendiendo al número de personas que debía mantener con interés.

Por lo anterior, se observa que la inclusión de la tecnología en el proceso formativo no debe darse al azar, en contraste, las competencias y habilidades digitales que el docente posea favorecerán o entorpecerán las estrategias que se diseñen. En este orden de ideas, existen iniciativas a nivel internacional que proponen métodos innovadores para motivar al alumnado, en este caso, las denominadas insignias digitales (Digital badges). Al respecto, Gibson et al. (2015) exponen que este es un mecanismo para explorar nuevas alternativas en la experiencia de la educación en línea. Parte de los beneficios que genera esta práctica es el incremento significativo del compromiso en el estudiante, contribuyendo así a la mejora en su desempeño. Los autores indican que, a través del reconocimiento público de estas insignias, el individuo puede visibilizar sus logros en la comunidad virtual, lo cual impacta positivamente en su rendimiento.

Hasta el momento, la revisión de la literatura apunta a que la inclusión de las TIC en los entornos de enseñanza y aprendizaje pueden modificar de manera importante la estructura tradicional. Por tal motivo, la inclusión de estas prácticas debe ir acompañada de una política pública coherente que involucre todos los niveles del sector educativo. Esta idea queda sustentada por Munro (2018) quien manifiesta que las estrategias para incorporar la tecnología en la educación superior desempeñan un factor determinante para su adecuada implementación. Fundamentado en el análisis de trece estrategias digitales aplicadas al proceso de enseñanza y aprendizaje establecidas por entidades públicas del Reino Unido durante 2003 y 2013, el autor expone que estas estrategias presentan en ocasiones contradicciones y dificultades importantes para su puesta en marcha, dada la multiplicidad de dimensiones que deben ser atendidas. Por tal razón, se podría explicar que los docentes el presente estudio tengan falencias en cuanto a la implementación en el aula, si no existen orientaciones claras sobre el cómo, por qué y para qué de estas herramientas.

Por último, las competencias relacionadas con las TIC para la productividad profesional, diseño de actividades con TIC y gestión de la información señalan que su incorporación en los procesos de enseñanza se realiza de manera regular, con rangos de respuestas importantes entre las categorías casi nunca y casi siempre. Asimismo, con la finalidad de profundizar en los datos obtenidos se realizó un análisis descriptivo utilizando medidas de tendencia central y posición (media, desviación típica, mínimo, máximo) para observar el comportamiento de los puntajes del cuestionario. La tabla 3, resume tales hallazgos.

La columna promedio refleja la media de los puntajes obtenidos para cada una de las competencias evaluadas en una escala del 1 al 5 , en donde 5 representa la mayor categoría (Siempre) y 1 la menor (Nunca). A su vez, el mínimo y máximo indican los valores extremos de las respuestas que suministraron los docentes y finalmente, la desviación típica (Dt) pone de manifiesto la variabilidad de las opiniones del grupo en cada competencia. Destaca de los datos analizados que la media obtenida está comprendida mayoritariamente entre los valores 4 y 5 , por tanto, se confirma que las categorías de respuesta más representativas en definitiva son siempre y casi siempre para todas las competencias a excepción de la Gestión de la información y el Diseño de actividades con TIC, las cuales arrojaron valores por debajo de 4 cayendo así en la categoría a veces. Por su parte, el rango de valores entre Min. 1 y Max. 5, señalan que hay diversidad de opiniones respecto a la frecuencia en la que aplican las competencias digitales; en el caso de aquellas con valores mínimos iguales a 3 se evidencia que al menos a veces se toman en cuenta estas competencias.

Tabla 3: Análisis descriptivo de puntajes de las competencias digitales

\begin{tabular}{|l|c|c|c|c|}
\hline \multicolumn{1}{|c|}{ Competencia } & Promedio & Mín. & Max & Dt \\
\hline Aplicación de las TIC & 4,50 & 2 & 5 & 1,28 \\
\hline Capacitación e información & 4,80 & 3 & 5 & 0,52 \\
\hline Gestión de entornos de aprendizaje & 4,35 & 1 & 5 & 1,39 \\
\hline Promoción de las TIC & 4,05 & 2 & 5 & 1,43 \\
\hline Gestión de la información & 3,90 & 1 & 5 & 1,02 \\
\hline $\begin{array}{l}\text { Promoción del uso ético, legal y seguro de las } \\
\text { TIC }\end{array}$ & 4,35 & 3 & 5 & 1,23 \\
\hline Diseño de actividades con TIC & 3,75 & 2 & 5 & 1,41 \\
\hline Actividades de Innovación TIC & 4,25 & 1 & 5 & 1,16 \\
\hline Ciudadanía digital & 4,40 & 1 & 5 & 1,05 \\
\hline TIC para la productividad profesional & 4,35 & 3 & 5 & 0,81 \\
\hline
\end{tabular}


Respecto a los valores de la desviación típica, que en la mayoría de los casos fue mayor a 1 (Dt>1), indican que las opiniones varían aproximadamente en una categoría en el grupo de docentes, sin embargo, existe menos dispersión de criterios en la competencia Capacitación e Información, lo que induce a pensar que hay mayor consistencia en la apreciación del grupo sobre la frecuencia en que aplican actividades relacionadas con tal dimensión.

\section{DISCUSIÓN Y ANÁLISIS FINAL}

Existe una alta autopercepción en los docentes referente a la aplicación de las competencias digitales evaluadas, sin embargo, minoritariamente en algunas de ellas se observó que a veces o incluso casi nunca o nunca se aplican; la que mayor falencia evidenció fue el Diseño de actividades con TIC. Esto pone de manifiesto la pertinencia de reflexionar activamente sobre las causas por las cuales se está limitando el uso de las TIC en los procesos de enseñanza desde la perspectiva de las competencias digitales.

Otro hallazgo relevante del análisis fue la poca variabilidad de las opiniones de los entrevistados respecto a su capacidad para mantenerse informados y en constante capacitación en temas de TIC para el ámbito educativo ( $\mathrm{Dt}=0.52$ ), señalando este hecho su compromiso por la formación que fomente la creatividad e innovación para estimular el aprendizaje en los estudiantes. Adicional a este hecho, las competencias digitales asociadas con el uso de las TIC para aumentar la productividad profesional, la promoción de su uso ético, legal y seguro y la capacitación e información revelaron que, en todos los casos los docentes las han aplicado al menos una vez, lo cual deja en evidencia que son tomadas en cuenta en todo momento en los procesos de enseñanza.

En el ámbito internacional, las competencias docentes han sido uno de los componentes clave para lograr procesos de enseñanza y aprendizaje acordes con las necesidades de la sociedad moderna. Como ejemplo de ello, la Comisión Europea (2018) así lo ha definido entre sus lineamientos declarándolo como un elemento esencial en la formación de los docentes. En este orden de ideas, es posible extraer como reflexión final que las instituciones educativas superiores enfrentan dos retos importantes, por una parte, incluir en los diferentes programas formativos el dominio de competencias digitales para los futuros profesionales, mientras que, a su vez implica reforzar el uso de la tecnología en todos los docentes que imparten la enseñanza a fin de lograr su integración total en el largo plazo.

Con base en los hallazgos resulta pertinente iniciar procesos de reflexión sobre el desarrollo actual que poseen los docentes en materia de competencias digitales en la educación superior, de este modo en concordancia con el propósito del presente artículo es preciso destacar que el acompañamiento en la formación de las TIC, apoyadas en TAC debe ser un compromiso conjunto, entre el docente e institución, que facilite la implementación de estas herramientas y forme a los estudiantes en temas de ciudadanía digital. Asimismo, es recomendable que dentro de los programas curriculares a nivel universitario se tomen en cuenta la capacitación en competencias digitales, más allá de una visión instrumental de aplicación, de tal forma que logre impactar significativamente en la productividad y práctica profesional del maestro.

\section{CONCLUSIONES}

Las TIC han provocado profundas transformaciones en diversos sectores de la sociedad, entre estos el educativo. En el proceso de enseñanza y aprendizaje la inclusión de herramientas tecnológicas ha facilitado la comunicación y comprensión de los numerosos contenidos que se imparten en la formación académica. Sin embargo, a pesar de estas ventajas, la inclusión de estos instrumentos supone un reto para los docentes, quienes han de adquirir las competencias y habilidades necesarias para poder implementarlas con éxito y que éstas se conviertan en una verdadera fuente de progreso. Tras la revisión de la literatura y con base en los hallazgos, se pueden extraer algunas ideas concluyentes: a) Es relevante emplear modelos probados y reconocidos para la gestión de las competencias digitales docentes, dado que esto no puede darse al azar; b) La formación en esta área debe estar articulada con políticas educativas emanadas del Estado y las instituciones de educación superior; c) Existen dificultades en el uso ético, legal y seguro de las TIC en los espacios formativos, por ello, hay que afianzar notablemente la capacitación en este ámbito; d) Los docentes requieren apoyo significativo en la gestión de la información y el diseño de actividades con TIC dado que, en algunos casos, no cuentan con los elementos necesarios para realizar llevar a cabo estos procesos de manera fluida.

\section{REFERENCIAS}

Badilla S., E., Descentralizar el Aprendizaje: Nuevos Retos para la Educación. Revista Electrónica Actualidades Investigativas en Educación, 7 (2007)

Banister, S. y R. Vannatta, Assessing NETS - T Performance in Teacher Candidates: Exploring the Wayfind teacher assessment; Journal of Digital Learning in Teacher Education, 29(2), 59-65 (2012) 
Bernard, R. M., y otros cinco autores, A meta-analysis of blended learning and technology use in higher education: From the general to the applied; Journal of Computing in Higher Education, 26(1), 87-122 (2014)

Buabeng A., C., Factors Influencing Teachers' Adoption and Integration of Information and Communication Technology into Teaching: A Review of the Literature; International Journal of Education and Development using Information and Communication Technology, 8(1), 136-155 (2012)

Bustos, A. y M. Román, La Importancia de Evaluar la Incorporación y el Uso de las TIC en Educación; Revista Iberoamericana de Evaluación Educativa, 4(2) (2016)

Cabero A., J., Formación del Profesorado Universitario en TIC. Aplicación del Método Delphi para la Selección de los Contenidos Formativos; Educación XXI, 17(1), 111-132 (2014)

Cacheiro G., M. L, Educación y Tecnología: Estrategias Didácticas para la Integración de las TIC. Editorial UNED Madrid, España (2018)

Colorado, A., B. L. y N. R. Edel, La Usabilidad TIC en la Práctica Educativa; Revista de Educación a Distancia, 30(2) (2012)

Domínguez, J. D. M., A. Domínguez, L. Acosta, M. Montenegro, H. Palma y Cardona, La función administrativa en la era de las TIC; Revista Lasallista de Investigación, 12(2), 139-151 (2015)

Enríquez, S., Luego de las TIC, LAS TAC; Ponencia presentada en las II Jornadas Nacionales de TIC e Innovación en el Aula, febrero (2012)

Ertmer, P. A., A. T. Ottenbreit L., y J. Tondeur, Teachers' Beliefs and Uses of Technology to Support 21st-Century Teaching and Learning; International handbook of research on teacher beliefs, 403 (2014)

Esteve M., F. M., La Competencia Digital Docente: Análisis de la Autopercepción y Evaluación del Desempeño de los Estudiantes Universitarios de Educación por Medio de un Entorno 3D; Doctoral dissertation, Universitat Rovira i Virgili, (2015)

European Commission, Digital Competence Framework for Educators (DigCompEdu) (en la web: https://ec.europa.eu/jrc/en/digcompedu, acceso: 15 de septiembre 2018), (2018)

European Commission, Evaluar la Competencia Digital Docente (en la web: https://goo.gl/ntzVga, acceso: 15 de septiembre 2018), (2018)

Expósito, E. O., E. M. Fuentes y J. R. Martínez, J, Aplicación de las TAC en un Entorno AICLE: Una Experiencia de Innovación en Educación Primaria; Aula de Encuentro, 19(1) (2017)

Gibson, D., N. Ostashewski, K. Flintoff, S. Grant y E. Knight, Digital badges in education; Education and Information Technologies, 20(2), 403-410 (2015)

Gil, J. J. S., Del TIC al TAC: Una aproximación al Modelado e Impresión 3D en Educación Superior; Rev Educ Cienc Salud, 14(1), 23-29 (2017)

Gisbert, M. y F. Esteve, Digital Leaners: la Competencia Digital de los Estudiantes Universitarios; La Cuestión Universitaria, (7), 48-59 (2016)

Gleason, B. y S. Von Gillern, Digital Citizenship with Social Media: Participatory Practices of Teaching and Learning in Secondary Education; Journal of Educational Technology \& Society, 21(1), 200-212 (2018)

Granados R., J. y otros cinco autores, Las Tecnologías de la Información y las Comunicaciones, las del Aprendizaje y del Conocimiento y las Tecnologías para el Empoderamiento y la Participación como Instrumentos de Apoyo al Docente de la Universidad del Siglo XXl; Medisur, 12(1) (2014)

Granados R., J., Las TIC, TAC, TEP, como Instrumento de Apoyo al Docente de la Universidad del Siglo XXI; Gestión Pedagógica con Uso TIC (2015)

Gutiérrez, L., Paradigmas Cuantitativo y Cualitativo en la Investigación Socio-educativa: Proyección y Reflexiones; Paradigma, 14(1y2), 7-25 (2017)

Hepp K., P., M. A. Prats y J. Holgado, Teacher Training: Technology Helping to Develop an Innovative and Reflective Professional Profile; International Journal of Educational Technology in Higher Education, 30-43 (2015)

Hernández S., R., C. Fernández y L. Baptista, Metodología de la investigación, 6ta ed., Mc Graw Hill, Ciudad de México, México (2014)

Howard, P., Paradigms of Research for the 21st Century: Perspectives and Examples from Practice; Journal of Professional, Continuing, and Online Education, 1(1) (2016)

Instefjord, E. J. y E. Munthe, Educating digitally competent teachers: A study of integration of professional digital competence in teacher education, Teaching and Teacher Education, 67, 37-45 (2017)

International Society for Technology in Education (ISTE), ISTE Standards Teachers (en la web: de https://goo.gl/HLDsu8, acceso: 15 de septiembre 2018), (2018)

Luz, C. G. M., Educación y Tecnología: Estrategias Didácticas para la Integración de las TIC. Editorial UNED (2018).

Marín, F. V., A. Inciarte, H. Hernández y R. Pitre, Estrategias de las Instituciones de Educación Superior para la Integración 
de las Tecnología de la Información y la Comunicación y de la Innovación en los Procesos de Enseñanza. Un Estudio en el Distrito de Barranquilla, Colombia. Formación universitaria, 10(6), 29-38 (2017)

Meza, J., O. Ortiz, S. Roman, J. Monguet y M. Tomala, The ICT enhancing the creativity through collective intelligence; ICST Trans. e-Education e-Learning, 4(14), e1 (2017)

Morphew, V. N., A Constructivist Approach to the National Educational Technology Standards for Teachers. International Society for Technology in Education (ISTE) (2012)

Munro, M., The complicity of digital technologies in the marketisation of UK higher education: exploring the implications of a critical discourse analysis of thirteen national digital teaching and learning strategies; International Journal of Educational Technology in Higher Education, 15(1), 11 (2018)

Niebles-Núñez, W. A., (2016). H. Hernández-Palma y D. Cardona-Arbeláez, Gestión tecnológica del conocimiento: herramienta moderna para la gerencia de instituciones educativas; Revista de Investigación, Desarrollo e Innovación, 7(1), 25-36 (2016)

Njoku, C., Information and Communication Technologies to Raise Quality of Teaching and Learning in Higher Education Institutions; International Journal of Education and Development using ICT, 11(1) (2015)

Otzen, T. y C. Manterola, Técnicas de Muestreo sobre una Población a Estudio; International Journal of Morphology, 35(1), 227-232 (2017)

Pérez, R. B., H. Palomares y F. DSilva, Cibercomunidad AVED. Experiencias de Integración Tecnológica para el Fomento y Desarrollo de la Educación A Distancia. Impacto de las Tecnologías en la Educación Superior a Distancia en Venezuela. Una década de retos, 113-128 (2017)

Peris, O. M. y J. Lindahl, Sustainable Learning in Higher Education. Springer, (2015)

Pinto S., A. R., P. Cortés y C. Alfaro, Hacia la Transformación de la Práctica Docente: Modelo Espiral de Competencias TICTACTEP; Pixel-Bit. Revista de Medios y Educación, (51) (2017)

Pontificia Universidad Javeriana - Cali /UNESCO, Competencias y Estándares TIC desde la Dimensión Pedagógica: Una Perspectiva desde los Niveles de Apropiación de las TIC en la Práctica Educativa Docente (en la web: https://goo.gl/ofL2hK, acceso: 15 de septiembre 2018), (2018)

Roblizo C., M. J. y G. Cózar, Usos y Competencias en TIC en los Futuros Maestros de Educación Infantil y Primaria: hacia una Alfabetización Tecnológica Real para Docentes. Pixel-Bit. Revista de Medios y Educación, 47, 23-39 (2015)

Siddiq, F., R. Scherer y J. Tondeur, Teachers' emphasis on Developing Students' Digital Information and Communication Skills (TEDDICS): A New Construct in 21st Century Education; Computers \& Education, 92, 1-14 (2016)

Stehling, V., U. Bach, A. Richert y S. Jeschke, Teaching professional knowledge to XL-classes with the help of digital technologies; Engineering Education 4.0, 77-90 (2016)

Stošić, L., The importance of Educational Technology in Teaching; International Journal of Cognitive Research in Science, Engineering and Education (IJCRSEE), 3(1), 111-114 (2015)

Svensson, M., y R. Baelo, Teacher students' perceptions of their digital competence, Procedia-Social and Behavioral Sciences, 180, 1527-1534 (2015)

Thomas, L. G. y D. Knezek, Information, Communications, and Educational Technology Standards for Students, Teachers, and School Leaders; At International Handbook of Information Technology in Primary and Secondary Education, pp 333348, Springer, Boston, MA (2008)

Tyner, K., Literacy in a Digital World: Teaching and Learning in the Age of Information, Routledge, (2014)

Uceda, J., S. Barro, F. Llorens y J. Franco, UNIVERSITIC 2010: Evolución de las TIC en el Sistema Universitario Español 2006-2010; Madrid: CRUE (2010)

Van Laar, E., A. Van Deursen, J. Van Dijk y J. De Haan, The relation between 21st-century skills and digital skills: $A$ systematic literature review; Computers in human behavior, 72, 577-588 (2017)

Venkatesh, V., A. Croteau y J. Rabah, J., Perceptions of Effectiveness of Instructional Uses of Technology in Higher Education in an Era of Web 2.0.; In System Sciences (HICSS), 2014 47th Hawaii International Conference, 110-119, enero, (2014)

Vivanco, G., Educación y Tecnologías de la Información y la Comunicación ¿Es Posible Valorar la Diversidad en el Marco de la Tendencia Homogeneizadora?; Revista Brasileira de Educação, 20(61) (2015) 